\title{
Statement from the International Collaboration on Endocarditis on the current status of surgical outcome in infective endocarditis
}

\author{
Andrew Wang \\ Duke University Medical Center, DUMC 3428, Durham, NC, USA \\ Correspondence to: Andrew Wang, MD. Professor of Medicine, Duke University Medical Center, DUMC 3428, Durham, NC 27710, USA. \\ Email: a.wang@duke.edu.
}

Submitted May 02, 2019. Accepted for publication May 22, 2019.

doi: 10.21037/acs.2019.05.19

View this article at: http://dx.doi.org/10.21037/acs.2019.05.19

Infective endocarditis (IE) continues to have a high inhospital mortality rate despite improvement in diagnosis and treatment. Surgery is an important, potentially lifesaving treatment, but clinical decision-making may be challenged by dilemmas such as (I) whether surgery will reduce mortality and morbidity of IE, with competing host factors as risks, and (II) the appropriate timing of surgical intervention, if indicated.

In general, the indications for surgery are IE complications that are not likely to be successfully treated or have a much higher risk of mortality if treated with antibiotic therapy alone. However, the predominance of studies that support surgical intervention in IE have been single center studies with retrospectively collected data and societal guidelines for IE management are overwhelmingly based on such observational studies $(1,2)$. Over the last 20 years, the International Collaboration on Endocarditis (ICE) has worked to improve the understanding and treatment of IE with the use of prospectively collected, multicenter data with standardized definitions.

In 1999, the ICE was launched as a merger of seven retrospective databases of IE from established IE centers in the United States, Great Britain, France, Spain and Sweden. The objective of the merged database was to evaluate regional differences in IE epidemiology. From this retrospective, merged database, 367 cases of definite prosthetic valve endocarditis (PVE) were identified, including $42 \%$ who had surgical intervention during their index hospitalization (3). The percentage of PVE patients treated with surgery was lower than expected, because PVE had been considered a strong indication for surgery with very high risk of treatment failure using antibiotic therapy alone. Using multivariable modeling, this study identified PVE characteristics associated with surgical treatment as well as those characteristics associated with in-hospital mortality. Patients who underwent surgery were matched with patients treated with antibiotic therapy only based on the propensity score for surgical intervention and there was no difference in in-hospital mortality among patients treated with surgery (3).

Our investigators were surprised by these results, because infection of a prosthetic valve had been considered a strong indication for surgery due to the high rate of complications and concerns about IE relapse if treated with antibiotics alone. Although notable differences existed in the clinical characteristics of IE patients who undergo surgery compared with those treated with antibiotics only, particularly, more IE complications such as heart failure and abscess, there was no statistically significant benefit for the sub-group treated with surgery (3).

In 2002, the ICE Prospective Cohort Study (ICE-PCS) was launched to expand the sample size and centers (23 centers in 15 countries) and to standardize data collection using modified Duke criteria for diagnosis and definitions for IE variables. This registry included 5,676 patients from 64 centers in 28 countries who were hospitalized between June 2000 and December 2006 for definite IE and who had vital status follow-up information available at 1 year after discharge; nearly half of patients had surgery performed during the index IE hospitalization. To adjust for the differences in surgically $v$ s. medically treated cases of IE, propensity analysis by inverse probability of treatment weighting was again used to compare patients with a similar propensity for surgery based on clinical, microbiologic 
and echocardiographic characteristics in addition to IE complications. In a study of native valve IE with both propensity adjustment and also instrumental variable analysis, we found that surgery was associated with lower in-hospital mortality for the overall cohort (absolute risk reduction: $-5.9 \%$ to $-11.2 \%$ ), with the strongest association in the patients with the most surgical indications (4). When surgery for prosthetic valve IE was evaluated in this registry for the outcome of 1-year mortality, similar results were found (5).

Because certain complications in IE have generally been considered strong indications or contraindications to early surgical treatment during the index hospitalization, other studies from the ICE-PCS sought to evaluate the association between surgery and mortality for many of these complications. Our studies have confirmed that surgery was strongly associated with lower risk of in-hospital and 1-year mortality among these high-risk sub-groups with heart failure or cardiac implanted electronic device infections $(6,7)$. On the other hand, surgery within 7 days of ischemic stroke was not associated with higher 1-year mortality compared with surgery after 7 days (8).

Although propensity adjustment for surgical treatment may attempt to control for selection bias, a remaining limitation in the ICE-PCS was absence of data related to operative risk. Operative risk models have been wellvalidated for many other cardiac conditions treated with surgery such as coronary artery disease and heart valve conditions; but these risk models had not been used in other studies of early surgery in IE. In order to prospectively evaluate the outcome of early surgery in IE, the ICE Plus registry collected prospective data on IE patients from 34 centers in 18 countries who were hospitalized between September 1, 2008, and December 31, 2012.

A total of 1,296 patients with left-sided IE were included. Surgical treatment was performed in $57 \%$ of the overall cohort at a median of 7 days from admission and in $76 \%$ of patients with a surgical indication (9). Among patients with a surgical indication who did not undergo surgery, reasons included poor prognosis, hemodynamic instability, death before surgery, stroke and sepsis. Although Staphylococcus aureus was the most common cause of IE, this causative organism was independently associated with a lower likelihood of surgery because of concomitant sepsis. We focused on 6-month mortality as the outcome measure, because of the recognized risk of surgery and long duration of medical therapy in patients with IE. Operative risk, as assessed by the Society of Thoracic Surgeon's IE (STS-
IE) score (10), was not associated with lack of surgery, but was strongly associated with 6-month mortality (9). In a recent separate study evaluating timing of surgery, there was no positive association between surgery within 7 days of admission or transfer and 6-month mortality (11).

Based on these many studies from three ICE registries, several important insights are evident. The benefit of surgery during the index hospitalization for IE is more robustly supported for cases with left-sided native or prosthetic IE complicated by heart failure, severe valvular regurgitation, abscess or large vegetation. However, the relationship between timing of surgery and outcome may be variable based on the specific surgical indication. Although $S$. aureus is the most common cause of IE and often associated with such IE complications, surgery is less often performed in these patients, likely related to adverse host factors such as hemodialysis. Although these observational studies with prospectively collected data have provided these conclusions, more randomized studies in intermediate risk IE patients are needed to evaluate the timing and outcome of surgery, including survival and other meaningful endpoints, particularly in patients with $S$. aureus infection.

\section{Acknowledgments}

None.

\section{Footnote}

Conflicts of Interest: The author has no conflicts of interest to declare.

\section{References}

1. Habib G, Lancellotti P, Antunes MJ, et al. 2015 ESC Guidelines for the management of infective endocarditis: The Task Force for the Management of Infective Endocarditis of the European Society of Cardiology (ESC). Endorsed by: European Association for Cardio-Thoracic Surgery (EACTS), the European Association of Nuclear Medicine (EANM). Eur Heart J 2015;36:3075-128.

2. Baddour LM, Wilson WR, Bayer AS, et al. Infective Endocarditis in Adults: Diagnosis, Antimicrobial Therapy, and Management of Complications: A Scientific Statement for Healthcare Professionals From the American Heart Association. Circulation 2015;132:1435-86.

3. Wang A, Pappas P, Anstrom KJ, et al. The use and effect of surgical therapy for prosthetic valve infective 
endocarditis: a propensity analysis of a multicenter, international cohort. Am Heart J 2005;150:1086-91.

4. Lalani T, Cabell CH, Benjamin DK, et al. Analysis of the impact of early surgery on in-hospital mortality of native valve endocarditis: use of propensity score and instrumental variable methods to adjust for treatmentselection bias. Circulation 2010;121:1005-13.

5. Lalani T, Chu VH, Park LP, et al. In-hospital and 1 -year mortality in patients undergoing early surgery for prosthetic valve endocarditis. JAMA Intern Med 2013;173:1495-504.

6. Athan E, Chu VH, Tattevin P, et al. Clinical characteristics and outcome of infective endocarditis involving implantable cardiac devices. JAMA 2012;307:1727-35.

7. Kiefer T, Park L, Tribouilloy C, et al. Association between valvular surgery and mortality among patients with infective endocarditis complicated by heart failure. JAMA

Cite this article as: Wang A. Statement from the International Collaboration on Endocarditis on the current status of surgical outcome in infective endocarditis. Ann Cardiothorac Surg 2019;8(6):678-680. doi: 10.21037/acs.2019.05.19
2011;306:2239-47.

8. Barsic B, Dickerman S, Krajinovic V, et al. Influence of the timing of cardiac surgery on the outcome of patients with infective endocarditis and stroke. Clin Infect Dis 2013;56:209-17.

9. Chu VH, Park LP, Athan E, et al. Association between surgical indications, operative risk, and clinical outcome in infective endocarditis: a prospective study from the International Collaboration on Endocarditis. Circulation 2015;131:131-40.

10. Gaca JG, Sheng S, Daneshmand MA, et al. Outcomes for endocarditis surgery in North America: a simplified risk scoring system. J Thorac Cardiovasc Surg 2011;141:98106.e1-2.

11. Wang A, Chu VH, Athan E, et al. Association between the timing of surgery for complicated, left-sided infective endocarditis and survival. Am Heart J 2019;210:108-16. 